\title{
Behavior modelling and sensing for machinery operations using smartphone's sensor data: A case study of forage maize sowing
}

\author{
Caicong $\mathrm{Wu}^{1,2}$, Zhibo Chen ${ }^{1}$, Dongxu Wang ${ }^{1}$, Zhihong $\mathrm{Kou}^{1}$, Yaping Cai ${ }^{3}$, Weizhong Yang $^{1 *}$ \\ (1. College of Information and Electrical Engineering, China Agricultural University, Beijing 100083, China; \\ 2. Key Laboratory of Remote Sensing for Agri-Hazards, Ministry of Agriculture and Rural Affairs, Beijing 100083, China; \\ 3. Department of Geography and Geographic Information Science, UIUC, Urbana-Champaign 61820, USA)
}

\begin{abstract}
Large-scale agricultural machinery cooperatives require technical statistic report of agricultural machinery operations to improve the efficiency of fleet management. This research proposed a smartphone-based solution to build the behavior model for agricultural machinery operations by using the embedded sensors including the GNSS, the accelerometer, and the microphone. The whole working process of agricultural machinery operation was divided into four stages: preparation, operation, U-turn, and transfer, each of which may contain the behaviors of stalling and idling. Field experiments were carried out by skilled operators, whose operations were typical agricultural machinery operations that could be used to extract behavior features. Butterworth low-pass filter was used to smooth the output from the accelerometer. Then, the operating data were collected through an APP when sowing the forage maize as a case study. Four stages of machinery operation can be preliminarily classified by using GNSS speed, while the identification of behaviors such as sudden acceleration and longtime idling that may increase fuel consumption, reduce machinery life, or decrease the working efficiency, requires extra information such as acceleration and sound intensity. The results showed that the jerk of accelerating can describe the severity of the sudden acceleration, the standard deviation of forward acceleration can reflect the smoothness of operation, the upward acceleration can be used to identify behaviors of stalling and idling, and the sound intensity during idling can capture the behavior of goosing the throttle. Further, the operating behavior figure can be drawn based on the above parameters. In conclusion, this research constructed several behavior models of agricultural machinery and operators by using smartphone's sensor data and established the base of the online assessing and scoring system for agricultural machinery operations.
\end{abstract}

Keywords: agricultural machinery operation, behavior modeling, smartphone, sensors, case study, forage maize DOI: $10.25165 /$ j.ijabe.20191206.4702

Citation: Wu C C, Chen Z B, Wang D X, Kou Z H, Cai Y P, Yang W Z. Behavior modelling and sensing for machinery operations using smartphone's sensor data: a case study of forage maize sowing. Int J Agric \& Biol Eng, 2019; 12(6): 66-74.

\section{Introduction}

Machinery cooperatives are the main carriers of agricultural machinery socialization service in $\mathrm{China}^{[1-3]}$. Large-scale cooperatives usually employ dozens of operators that lead to more complicated management relations than family farms. In order to improve fuel consumption, machinery life, and work efficiency, it is necessary to strengthen the fleet management during agricultural machinery operations. Currently, GNSS ${ }^{[4-6]}$ and ISOBUS ${ }^{[7,8]}$ based fleet management are widely used and can realize real-time visibility of vehicle location, status, and diagnostics. However, it cannot record subtle but important operating behaviors ${ }^{[9]}$, such as longtime stalling or idling, sudden acceleration, sharp turning, and

Received date: 2018-09-09 Accepted date: 2019-10-15

Biographies: Caicong $\mathbf{W u}, \mathrm{PhD}$, Associate Professor, research interest: location-based service of farm machinery, Email: wucc@cau.edu.cn; Zhibo Chen, $\mathrm{PhD}$ candidate, research interest: location-based service of farm machinery, Email: bobodi@qq.com; Dongxu Wang, Master candidate, research interest: location-based service of farm machinery, Email: dongxu_wang@ cau.edu.cn; Zhihong Kou, Master candidate, research interest: location-based service of farm machinery, Email: 603040848@qq.com; Yaping Cai, PhD candidate, research interest: mobile information collection, Email: caicai_pku@ 163.com.

*Corresponding author: Weizhong Yang, $\mathrm{PhD}$, Associate Professor, research interest: location-based service of farm machinery. College of Information and Electrical Engineering, China Agricultural University, No.17 Qinghua East Road, Haidian District, Beijing 100083, China. Email: ywz@cau.edu.cn. etc. ${ }^{[10]}$. The main reason is that GNSS based telematics terminals cannot capture the transient data of operating behaviors during operation $^{[11,12]}$. Therefore, extra information from external sensors are required to detect those subtle but important operating information of agricultural machinery.

Smartphones have been rapidly developed and widely used nowadays. In terms of behavior detection, there are many smartphone-based researches using the high frequency transient data captured by embedded sensors. By placing the smartphone in a relatively fixed position (such as in a pocket) of an operator, some features such as the size and the frequency of the wave peaks and the roughness, can be recorded with the tri-axial accelerometer ${ }^{[13-16]}$. Further, using the machine-learning classifiers such as SVM, decision tree, and neural network, can classify and identify different behaviors including stalling, walking, running, and etc., which can achieve a recognition accuracy about $95 \%{ }^{[17,18]}$. When combined with geographic information, the method is widely used in caring for the old and the young, and for sports pattern recognition ${ }^{[19-22]}$. In the field of automobile driving, accelerometers, and gyroscopes are widely used in driving style and driving safety recognition ${ }^{[23-25]}$, such as sudden acceleration, sudden braking, speeding, and sharply turning ${ }^{[24,26,27]}$. These behaviors can be identified according to the features of the accelerations and the change of angles ${ }^{[24]}$. For agricultural machinery fault diagnosis ${ }^{[28]}$, some researchers used micro-electromechanical systems (MEMS)-based sensors to identify several induction motor failures ${ }^{[29]}$. Accelerometers have 
also been used to assess the efficiency of tractor transmissions ${ }^{[30]}$.

In this paper, a smartphone-based solution was proposed to detect the agricultural machinery operation and to identify the details of the operators' behaviors. Smartphone' sensors were used to collect the experimental data of agricultural machinery operations and the features were extracted to construct the behavior recognition models. Finally, the operators' behaviors were identified using the data of forage maize sowing, and the working behavior figures were drawn as a technical statistics report of the field production.

\section{Material and method}

\subsection{Technical route}

An investigation was conducted in three large-scale agricultural machinery cooperatives in Beijing to determine the most valuable behaviors that cooperative managers concerned about. Then features were extracted and behavior models were developed through field experiments. Finally, a case study was conducted using the data of the forage maize sowing. Obviously, different agricultural machinery operations have different behavior features and management requirements.

Data processing mainly includes three steps: filtering the acceleration, segmenting the trajectories, and identifying the behaviors. Segmenting the trajectories is to roughly divide the trajectories into several groups based on different time periods. Then the specific behaviors can be identified through adding the extra features of acceleration and sound intensity. The processing procedures are shown in Figure 1.

\subsection{Behavior and sensors}

Figure 2 shows four main stages including preparation, operation, U-turn, and transfer of in-field agricultural machinery operation. For each stage (see Table 1), the related operating behaviors were further defined and the required smartphone's sensors were selected. For most of the operating behaviors, both the GNSS and the accelerometer were used. As shown in Table 2, the operating behavior of stalling and idling may exist in all working stages. Therefore, these two behaviors would be detected after the detection of operating behaviors in the Table 1.

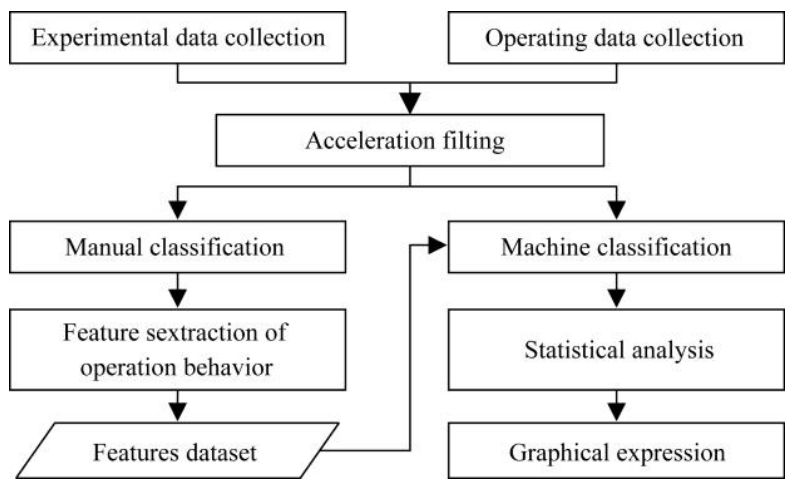

Figure 1 Technical route of data processing

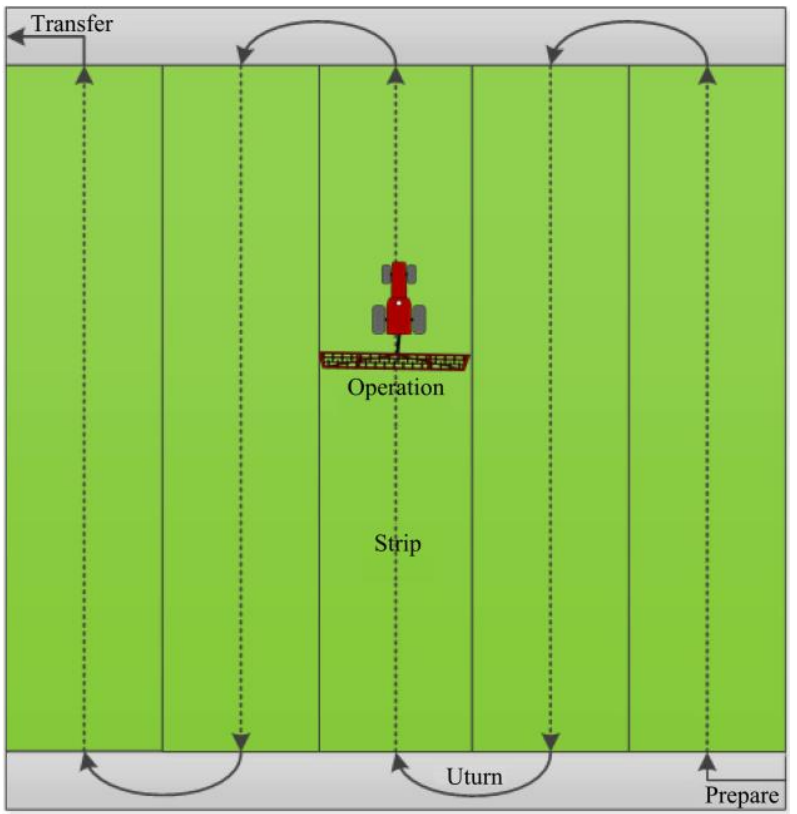

Figure 2 Four stages of in-field machinery working process

Table 1 Working stages, behaviors, and required sensors for in-field machinery operation monitoring

\begin{tabular}{|c|c|c|c|}
\hline Working stage & Behavior & Description & Sensors \\
\hline \multirow{2}{*}{ Preparation } & Preparing & Preparation time before operating & GNSS \\
\hline & Goosing & Goosing the throttle to preheat the engine & GNSS, accelerometer, microphone \\
\hline \multirow{6}{*}{ Operation } & \multirow{2}{*}{ Accelerating } & Sudden acceleration & GNSS, $y$-axis accelerometer \\
\hline & & Acceleration time & GNSS, $y$-axis accelerometer \\
\hline & \multirow{3}{*}{ Operating } & Rate of optimum operating speed & GNSS \\
\hline & & Smoothness & $y$-axis accelerometer \\
\hline & & Continuity & GNSS, tri-axial accelerometer \\
\hline & Braking & Sudden deceleration & GNSS, $y$-axis accelerometer \\
\hline \multirow{2}{*}{ U-turn } & \multirow{2}{*}{ U-turn } & Time range of U-turn & GNSS, tri-axial accelerometer \\
\hline & & STD of U-turn & GNSS, tri-axial accelerometer \\
\hline Transfer & Transferring & Time range of transferring & GNSS \\
\hline
\end{tabular}

Table 2 Behavior of stalling and idling and required sensors

\begin{tabular}{cccc}
\hline Working stage & Behavior & Description & Sensors \\
\hline \multirow{2}{*}{ All stages } & Stalling & Time of stalling & GNSS, tri-axial accelerometer \\
& Idling & Time of idling & GNSS, tri-axial accelerometer \\
\hline
\end{tabular}

\subsection{Data acquisition}

GNSS and sensors data were collected for the extraction of standard operating behavior features through the APP developed by the authors from both the regular field experiments and the forage maize sowing in June of 2018 for the case study.

\subsubsection{APP for data collection}

As shown in Figure 3a, an Android-based APP was developed to obtain the smartphone's sensors data. The sampling frequency 
was $10 \mathrm{~Hz}$ and the data were stored in the smartphone when sampling. The following variables were collected:

$$
D=\left\{\left(d, t, \text { lon, lat }, v, h, a_{x}, a_{y}, a_{x}\right)\right\}_{i=1}^{N}
$$

where, $D$ is the dataset, $d$ is the date; $t$ is the Beijing time; lon is the GNSS longitude, $\left({ }^{\circ}\right)$; lat is the GNSS latitude, $\left({ }^{\circ}\right) ; v$ is the GNSS speed, $\mathrm{m} / \mathrm{s} ; h$ is the GNSS heading direction, $\left({ }^{\circ}\right) ; a$ is the acceleration of the related direction $(\mathrm{x}, \mathrm{y}, \mathrm{z}), \mathrm{m} / \mathrm{s}^{2}$; and $N$ represents the number of sampling information instants.

Sound was recorded by an independent APP. Smartphones used in this research were Huawei B199, which were located directly above the rear wheel of the tractor and placed horizontally (see Figure 3b). Figure $3 \mathrm{c}$ shows the phone coordinate system: $x$-axis from left to right, $y$-axis from bottom to top, and $z$-axis from inside to outside. The $y$-axis is parallel to the forward motion of the tractor, while $z$-axis is facing up towards the sky.

\subsubsection{Field experiments}

In the field (Figure 4a), the skilled operators performed the behaviors of accelerating, operating, braking, and etc., which simulated the normal operating behaviors and the abnormal operating behaviors, respectively, that could be used to extract the operating behavior features. The agricultural machinery used for field test were John Deere 1204 and John Deere planters.

\subsubsection{Field operations}

Operating data was collected on June $16^{\text {th }}, 2018$, in two fields as shown in Figure 4b. Tractors and implements mentioned above were used for sowing (Figure 4c). On September $3^{\text {rd }}, 2018$, the authors returned to the two fields (Figure 4d) to observe the growth in order to verify the results of trajectory segmentation.
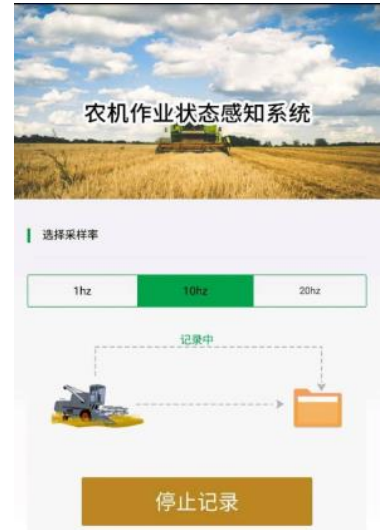

a. APP for data collection

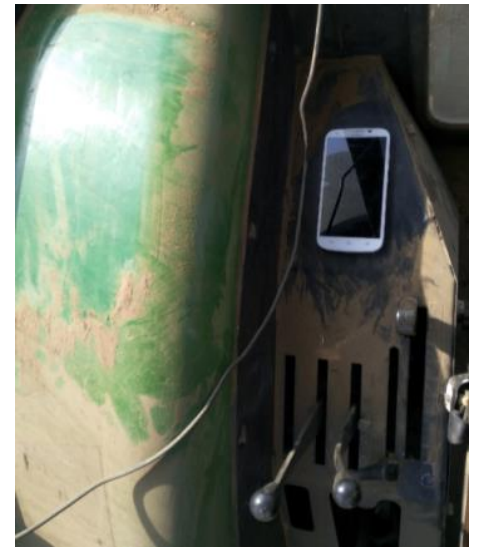

b. Placement of smartphone on the tractor

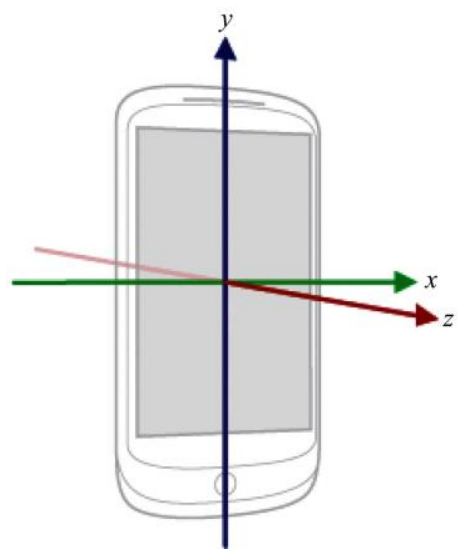

c. Smartphone coordinate system

Figure 3 Phone coordinate system and placement position of smartphone data processin

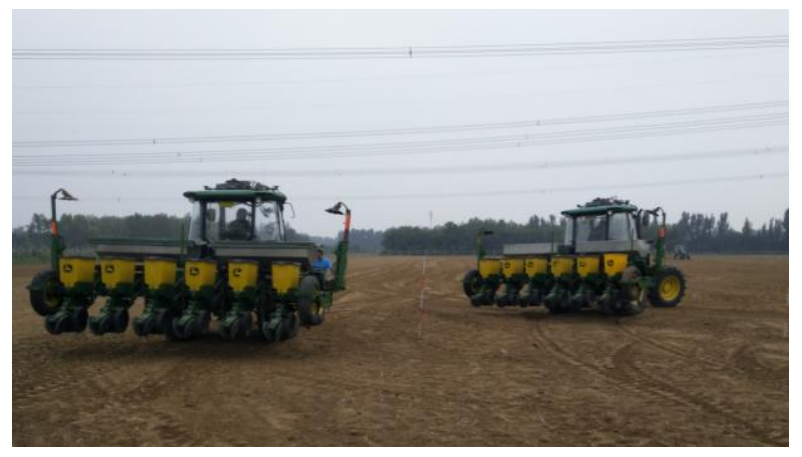

a. Field experiments

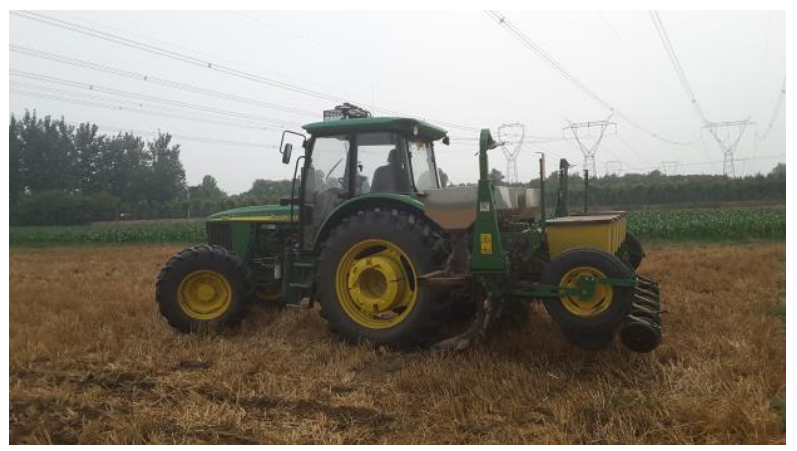

c. Forage maize plantation

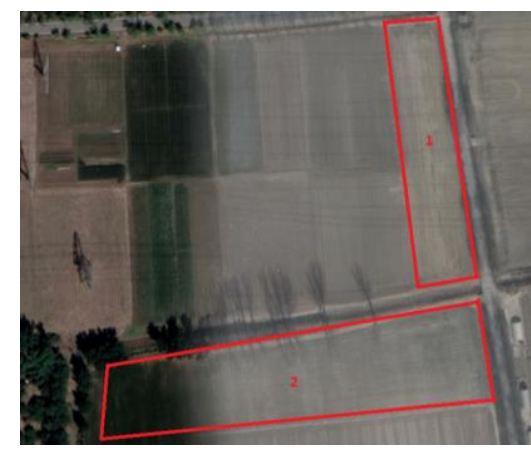

b. Two fields for forage maize plantation

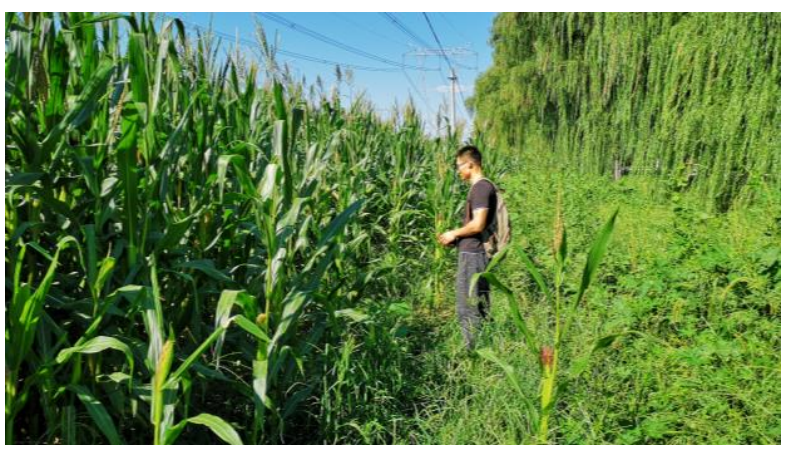

d. Inspection and confirmation

Figure 4 Field experiments and data collections combined with forage maize plantation

\subsection{Data processing}

Data processing program was developed using Matlab 2018a.

\subsubsection{Acceleration filtering}

Butterworth low-pass filter was used for acceleration filtering ${ }^{[31]}$. The parameters of the filter are as followings:

$$
\text { Filter }:\left\{\begin{array}{l}
W_{p}=2 /\left(F_{s} / 2\right) \\
W_{s}=4 /\left(F_{s} / 2\right) \\
R_{p}=2 \\
R_{s}=40 \\
F_{s}=10
\end{array}\right.
$$


where, $W_{p}$ and $W_{s}$ are the pass-band and the stop-band edges respectively; $F_{s}$ is sampling frequency; $R_{p}$ is the allowable decibels of ripple; and $R_{s}$ is the minimum attenuation in the stop.

2.4.2 Segmenting trajectory

A notable feature of sowing is that during the startup and the end of machinery operation, in order to drop and lift the planter, there is a short but significant stopping, which means the smartphone' GNSS sensor can obtain some zero speed trajectories. Therefore, the trajectories can be segmented by the following steps:

(1) Find the ids of the trajectory with zero speed.

(2) Combine those working trajectories with nonzero speed (part of them should be the operating trajectory).

(3) Identify the behaviors using the operating behavior model developed based on the extracted operating features.

\subsection{Behavior modeling}

\subsubsection{Preparation}

The trajectories before operating in the operation strips can be defined as the preparation trajectories. Thus, when the first operation strip is detected, previous trajectories will be classified as the preparation trajectories. The time range of preparation is used to describe whether the preparation is sufficient before field operation. Longtime preparation, especially longtime idling during preparation, should be avoided.

\subsubsection{Operation}

Figure 5 shows a schematic diagram of one entire agricultural machinery operation, which mainly includes three phases, i.e. accelerating, operating, and decelerating. The left $y$-axis is the acceleration, and the right $y$-axis is the speed. Operating is the core phase during the entire agricultural machinery operation, whose behavior is mostly concerned by the cooperative managers.

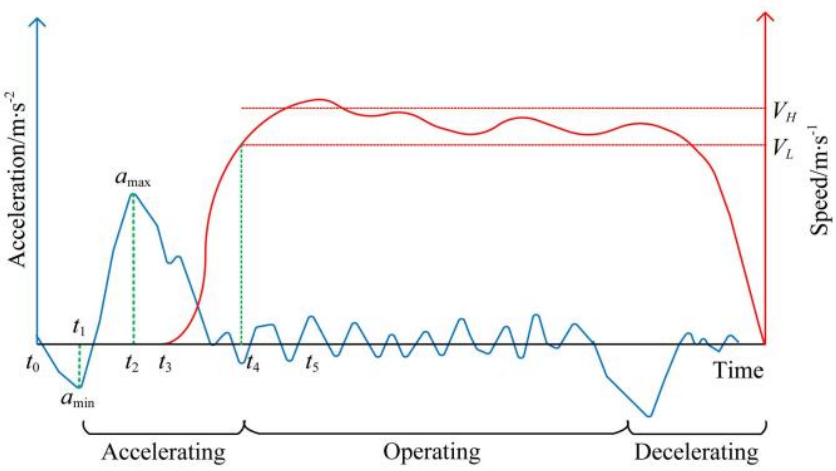

Figure 5 Schematic diagram of y-axis acceleration and GNSS speed in operation stage

(1) Accelerating

In the startup phase of the field operation, seeding opener gradually enters the tillage soil with increasing soil resistance. At the meantime, the operator can make the tractor reach desired working speed within a certain period of time by slamming on the throttle with increasing engine output to offset the soil resistance. The appropriate acceleration is encouraged to output the proper power and keep the healthy of agricultural machinery.

i) Sudden acceleration

Sudden acceleration is a bad driving behavior. Taking off like a shot not only spends more gas than gradually accelerating, but also is bad for the engine. The following formula and the parameter (jerk) are constructed to detect the behavior of sudden acceleration.

$$
\text { jerk }=\frac{a_{\max }-a_{\min }}{t_{2}-t_{1}}
$$

where, $a_{\max }$ and $a_{\min }$ are the maximum and the minimum values of the forward acceleration in the startup phase respectively; $t_{1}$ and $t_{2}$ represent the moments corresponding to $a_{\min }$ and $a_{\max }$ (see Figure 5), respectively. Obviously, the harder the throttle, the larger the jerk.

Sudden acceleration usually occurs within $3 \mathrm{~s}$ before and after the trajectories with zero speed, so the data processing program was developed to find the moment of the maximum forward acceleration and the minimum forward acceleration.

ii) Acceleration time

In contrast to the slamming on the throttle, some operators may start slowly, and the agricultural machinery need more time to reach the desired working speed than the standard operation, which may also decrease the operation quality. Assuming the optimum working speed range is $\left[V_{L}, V_{H}\right]$. The rate of acceleration time $\left(R_{02 L}\right)$ reaching $v_{L}$ from zero speed can be calculated as following:

$$
R_{02 L}=\frac{T_{4}-T_{3}}{T_{S}} \times 100 \%
$$

where, $T_{S}$ is the working time for the related strip; $T_{3}$ is the startup time; and $T_{4}$ is the moment when machinery's speed reaches $V_{L}$.

\section{(2) Operating}

After accelerating, the machinery enters the operating process. The mean and the standard deviation of the operating velocity were used to extract the trajectory of the operation. It is assumed that the operating velocity would keep stable during the operation. The recognition model of operating behavior is the followings.

$$
C_{1}(O)=\left\{\begin{array}{l}
\bar{v} \leq M A X_{-} A V G_{-} O P E R \_V E L\left(\bar{V}_{H}\right) \\
\bar{v} \geq M I N_{-} A V G_{-} O P E R \_V E L\left(\bar{V}_{L}\right) \\
\delta(v) \leq O P E R \_V E L \_S V\left(V_{S V}\right) \\
T_{s} \geq M I N_{-} O P E R_{-} T I M E \\
T_{s} \leq M A X_{-} \text {OPER_TIME }
\end{array}\right.
$$

In the above classifier for operation identification, the limitations for operating time were used to exclude some U-turn and transfer trajectories. Therefore, more information regarding the operation before data processing is needed to improve the segmentation efficiency and accuracy.

i) Working in optimum speed

The rate of the optimum working speed $\left(R_{L 2 H}\right)$ represents how much time $\left(\Delta T_{L 2 H}\right)$ the planter needs to work in the speed range of $\left[v_{L}, v_{H}\right]$, which can be an indicator of the performance of the planter. $R_{L 2 H}$ is calculated by the following formula.

$$
R_{L 2 H}=\frac{\Delta T_{L 2 H}}{T_{S}} \times 100 \%
$$

ii) Over speed

The operation of the over speed would decrease the sowing quality. The rate of over speed $\left(R_{O S}\right)$ reflects the over speed condition during the operating phase.

$$
R_{O S}=\frac{\Delta T_{O S}}{T_{S}} \times 100 \%
$$

where, $\Delta T_{O S}$ is the length of time of over speed.

iii) Smoothness

The positioning frequency of GNSS is usually $0.1-1 \mathrm{~Hz}$, which cannot capture the subtle forward speed changes. Therefore, the standard deviation of y-axis acceleration $\left(\sigma_{y a}\right)$ is used to reflect the smoothness of the operating.

$$
\sigma_{y a}=\sqrt{\frac{1}{N} \sum_{i=1}^{N}\left(a_{i}-\bar{a}_{y}\right)^{2}}
$$

where, $\bar{a}_{y}$ is the mean of forward acceleration.

iv) Continuity

Good working behavior is to complete the task in one go. If the work is interrupted many times, it will decrease both the 
operation quality and operation efficiency. With the speed-based segmentation, this research can accurately detect the interruptions.

\subsubsection{U-turn}

In general, as shown in Figure 2, the U-turn trajectory is the trajectory between two operation strips. Therefore, by combining the trajectories between two operation strips, a complete U-turn trajectory can be obtained. Obviously, longtime U-turn should be avoided as much as possible. If a long duration of U-turn happens, the behavior of stalling or idling should be detected.

2.5.4 Transfer

When the final operation strip completed, the trajectories after the operating and before leaving the field is defined as transfer phase. In general, operators will go to the next field or return home, but the situation that the operator subjectively deliberates stay in the field cannot be ruled out. If a long duration of transfer happens, the behavior of stalling or idling should be also detected.

\subsubsection{Stalling, idling, and goosing}

Longtime stalling or idling reflects insufficient preparation or slack during the work. When the behavior of stalling or idling happens, they cannot be distinguished only by the GNSS speed $\left(v_{\mathrm{GNSS}}=0\right)$; however, they can be distinguished by the mean or the standard deviation of the tri-axial acceleration or the sole upward acceleration. When agricultural machinery is completely stalling, it has no vibration, its mean or standard deviation is extremely small. While when agricultural machinery is idling, the engine causes slight vibrations, and the mean or the standard deviation is greater than that of stalling but less than that of the operating.

i) Identification of stalling and idling

The behaviors of stalling and idling are identified by the following classifier:

$$
C_{2}(W)=\left\{\begin{array}{llll}
\text { stalling } & \bar{V}=0 & \& & \delta(a)<\delta(a)_{s o i} \\
\text { idling } & \bar{V}=0 & \& & \delta(a) \geq \delta(a)_{\text {soi }}
\end{array}\right.
$$

where, $\delta(a)$ is standard deviation of the tri-axial acceleration; and $\delta(a)_{s o i}$ is the threshold to distinct stalling and idling (see Table 4).

ii) Identification of idling and goosing

In order to preheat the tractor as soon as possible, some operators usually heat the engine by goosing the throttle after startup. Goosing the throttle is a bad behavior. It is impossible to detect this behavior by only using GNSS speed and the upward acceleration. However, it can be captured through smartphone' microphone, since goosing the throttle will inevitably increase sound intensity of the environment and sharp peaks of sound intensity would appear for a short time. Goosing the throttle during idling state is judged by sound intensity as following formula.

$$
C_{3}(G)=\left\{\begin{array}{l}
\text { idling } \quad \bar{V}=0 \quad \& \quad \delta(i)<\delta(i)_{i o g} \\
g o o \sin g \quad \bar{V}=0 \quad \& \quad \delta(i) \geq \delta(i)_{i o g}
\end{array}\right.
$$

where, $\delta(i)$ is the standard deviation of the sound intensity; $\delta(i)_{i o g}$ is the threshold to distinct idling and goosing (see Table 4).

\section{Results and discussions}

\subsection{Features of behaviors}

Through the field experiments, the waveform, mean, and standard deviation of tri-axial acceleration of stalling, idling, goosing, and operating can be obtained (Figure 6). As Figure 6a shows, the waveforms of different operating behaviors have significant differences.

Figure $6 \mathrm{~b}$ shows the time usage of the above behaviors. Behaviors of stalling, idling, and operating can be easily classified using the mean and standard deviation of the tri-axial acceleration. To distinguish between idling and goosing, the sound intensity was recorded through the smartphone microphone. In Figure 7, $y$-axis represents the normalized value of the sound intensity, with range between -1 and 1 . The stalling state has a very low sound intensity. The sound intensity of idling state is large, whose mean value reaches $0.09 \mathrm{w} / \mathrm{m}^{2}$, while the sound intensity of goosing is larger, whose peak value reaches $0.19 \mathrm{w} / \mathrm{m}^{2}$. Therefore, it is feasible to distinguish between the behaviors of idling and goosing through the microphone.

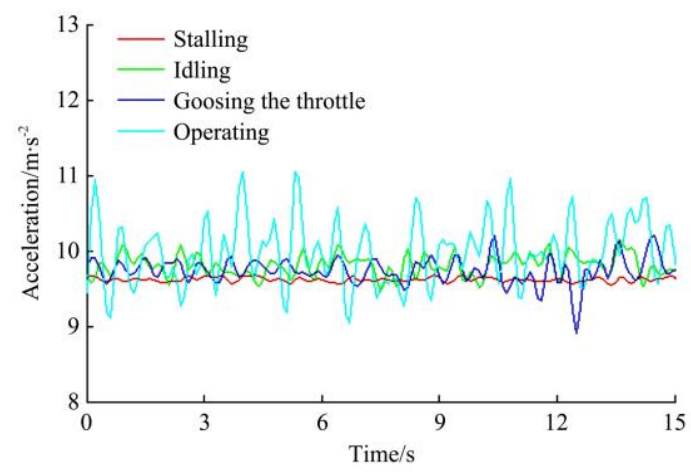

a. Tri-axial acceleration waveform

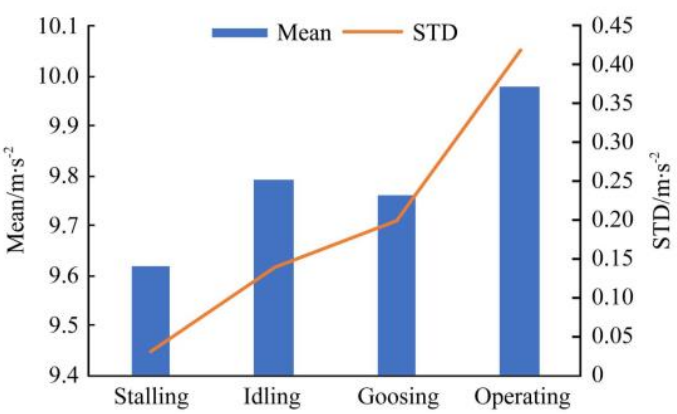

b. Mean and STD of Tri-axial acceleration

Figure 6 Tri-axial acceleration of stalling, idling, goosing the throttle, and operating

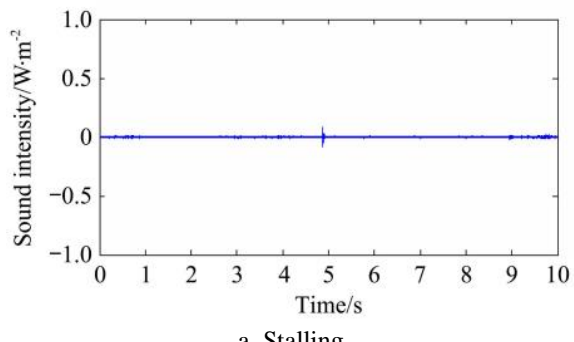

a. Stalling

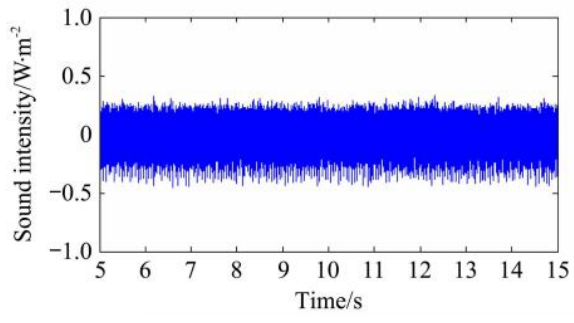

b. Idling

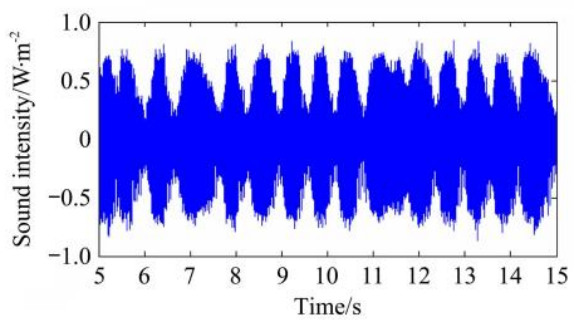

c. Goosing the throttle

Figure 7 Sound intensity waveform of stalling, idling, and goosing the throttle states 
Since the sound file is much larger than that of other sensors, the sound cannot be recorded all the time. Since goosing the throttle usually happens during the preparation stage, only the sound before the operating stage will be recorded.

Based on the above analysis, the basic features were extracted to describe the behaviors of machinery and operators (Table 3). Main parameters include the mean of speed, the mean and the standard deviation of tri-axial acceleration, and the sound intensity.

Table 3 Features of machinery behaviors

\begin{tabular}{|c|c|c|c|c|}
\hline \multirow{2}{*}{ Status } & \multirow{2}{*}{$\frac{\text { Speed }}{\text { Mean } / \mathrm{km} \cdot \mathrm{h}^{-1}}$} & \multicolumn{2}{|c|}{ Tri-axial acceleration } & \multirow{2}{*}{$\begin{array}{c}\text { Sound } \\
\text { intensity } / \mathrm{W} \cdot \mathrm{m}^{-2}\end{array}$} \\
\hline & & Mean $/ \mathrm{m} \cdot \mathrm{s}^{-2}$ & $\mathrm{STD} / \mathrm{m} \cdot \mathrm{s}^{-2}$ & \\
\hline Operation & $>0$ & 9.97 & 0.42 & 0.16 \\
\hline U-turn & $>0$ & 9.99 & 0.67 & 0.08 \\
\hline Transfer & $>0$ & 10.00 & 0.55 & 0.07 \\
\hline Stalling & 0 & 9.62 & 0.03 & $<0.01$ \\
\hline Idling & 0 & 9.79 & 0.14 & 0.09 \\
\hline Goosing & 0 & 9.76 & 0.20 & 0.19 \\
\hline
\end{tabular}

\subsection{Thresholds for behavior identification}

The parameters (Table 4) for trajectory segmentation involving the empirical knowledge of cooperative managers were defined. For instance, to identify the behavior of idling and goosing, the average of their features $\left(0.14 \mathrm{~m} / \mathrm{s}^{2}\right.$ and $0.20 \mathrm{~m} / \mathrm{s}^{2}$ in Table 3$)$ were used as the threshold $\left(0.17 \mathrm{~m} / \mathrm{s}^{2}\right.$ in Table 4$)$.

Table 4 Thresholds for behavior identification

\begin{tabular}{cccc}
\hline Reference value & Threshold & Unit & Description \\
\hline $\bar{V}_{H}$ & 7.7 & $\mathrm{~km} \cdot \mathrm{h}^{-1}$ & $\begin{array}{c}\text { The upper limit of average operation } \\
\text { speed for detection }\end{array}$ \\
$\bar{V}_{L}$ & 4.5 & $\mathrm{~km} \cdot \mathrm{h}^{-1}$ & $\begin{array}{c}\text { The low limit of average operation } \\
\text { speed for detection }\end{array}$ \\
$V_{C V}$ & 20 & $\%$ & $\begin{array}{c}\text { The coefficient of variation of } \\
\text { operation speed for detection }\end{array}$ \\
$\delta(a)_{\text {soi }}$ & 0.095 & $\mathrm{~m} \cdot \mathrm{s}^{-2}$ & $\begin{array}{c}\text { Standard deviation of acceleration to } \\
\text { distinct stalling and idling }\end{array}$ \\
$\delta(i)_{\text {iog }}$ & 0.17 & $\mathrm{~W} \cdot \mathrm{m}^{-2}$ & $\begin{array}{c}\text { Standard deviation of sound intensity } \\
\text { to distinct idling and goosing }\end{array}$ \\
$V_{H}$ & 7.0 & $\mathrm{~km} \cdot \mathrm{h}^{-1}$ & $\begin{array}{c}\text { The upper limit of optimum operation } \\
\text { speed interval }\end{array}$ \\
$V_{L}$ & 6.0 & $\mathrm{~km} \cdot \mathrm{h}^{-1}$ & $\begin{array}{c}\text { The low limit of optimum operation } \\
\text { speed interval }\end{array}$ \\
\hline
\end{tabular}

\subsection{Case study}

\subsubsection{Whole process}

Figure 8 shows the statistics of time consuming of four stages in field I and field II. Taking Figure 8a as an example, the operator in field I accounts for $32 \%$ of the operating time, and the U-turn time accounts for $43 \%$, while the preparation and transfer time accounts for $10 \%$ and $16 \%$. It can be found that the effective working time of the agricultural machinery in the field is very limited. This simple result shocked the cooperative managers, who did not expect such inefficiency. After all, the effective operation time is only about two fifths, and equivalent time is used for U-turn. The main reason is that the length of field is usually less than $400 \mathrm{~m}$ in Beijing. Therefore, how to design U-turn mode and to improve the operator's U-turn skill is very important.

3.3.2 Technical statistics

Based on the methods mentioned above, trajectory segmentation and behavior recognition were performed for the two fields, and the technical records of field I are shown in Figure 9. The blue line and the black line represent the operation and the U-turn. The red circle, the blue circle, and the yellow circle represent jerk of acceleration, time of operation, and time of U-turn, respectively. In order to make the figure clear, two east-west strips in both sides of the field were hided.
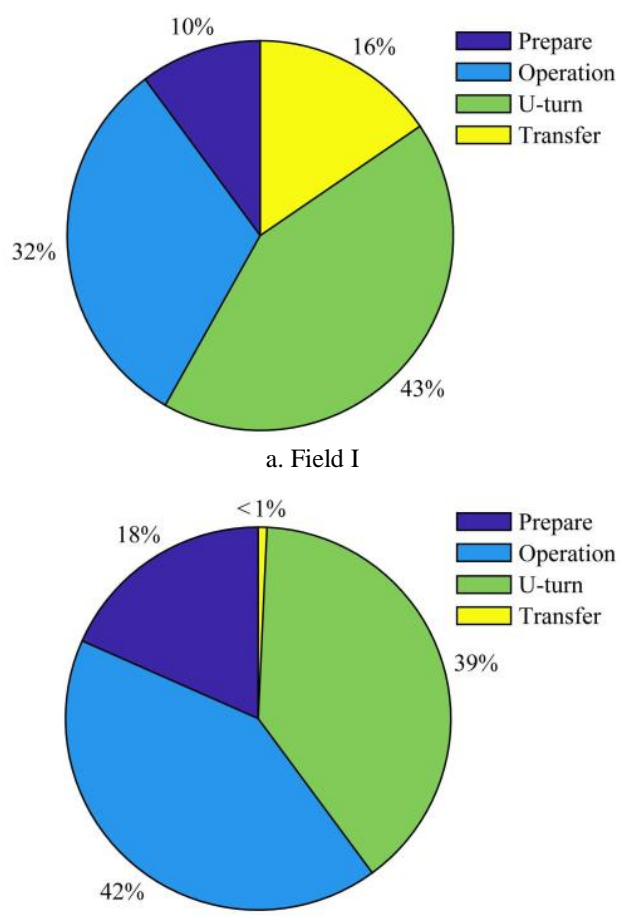

b. Field II

Figure 8 Statistical analysis of time spent in the field

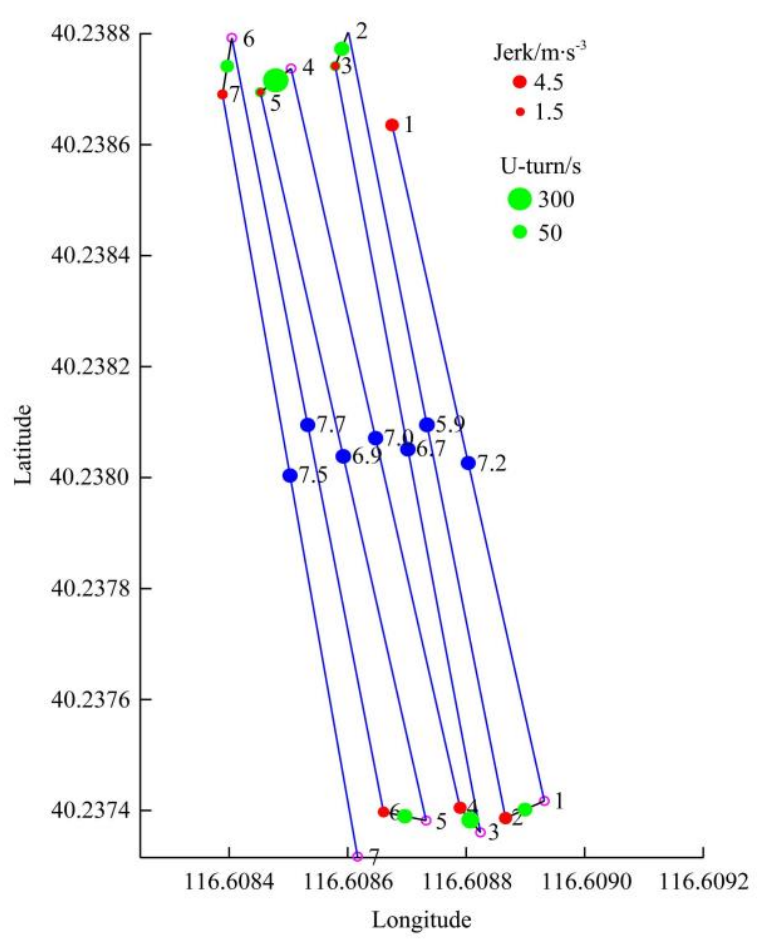

Figure 9 Operating behavior pictures of filed II

(1) Startup

The starting jerk for each strip and the time of acceleration can be obtained. As shown in Table 5, in general, the larger the jerk, the shorter the time to reach $V_{L}$.

Figure 10 shows the acceleration of four operation strips in the starting phase. The accelerator pedaling force can be reflected by the change of acceleration. S1, S7, and S5 reflect fast, medium, and slow acceleration, respectively. The large jerk means that the machinery can be brought to the desired speed faster and play the 
best performance of the planter. Table 5 lists $R_{02 L}$ for each strip. The jerks of S1 and S7 are relatively large, reaching $4.35 \mathrm{~m} / \mathrm{s}^{3}$ and $2.41 \mathrm{~m} / \mathrm{s}^{3}$, respectively, while $R_{02 L}$ is $9.2 \%$ and $15.1 \%$. When jerk of the $\mathrm{S} 3$ is only $1.3 \mathrm{~m} / \mathrm{s}^{3}, R_{02 L}(22.4 \%)$ is large.

However, the jerk monitored in the paper is a short-term behavior, it is not proportional to the time of acceleration. For instance, when $j e r k_{\mathrm{s} 2}=4.07$, its $R_{02 L}$ is $32.36 \%$, and the time of acceleration is still longer than that of S3 due to the failure to continue accelerating.
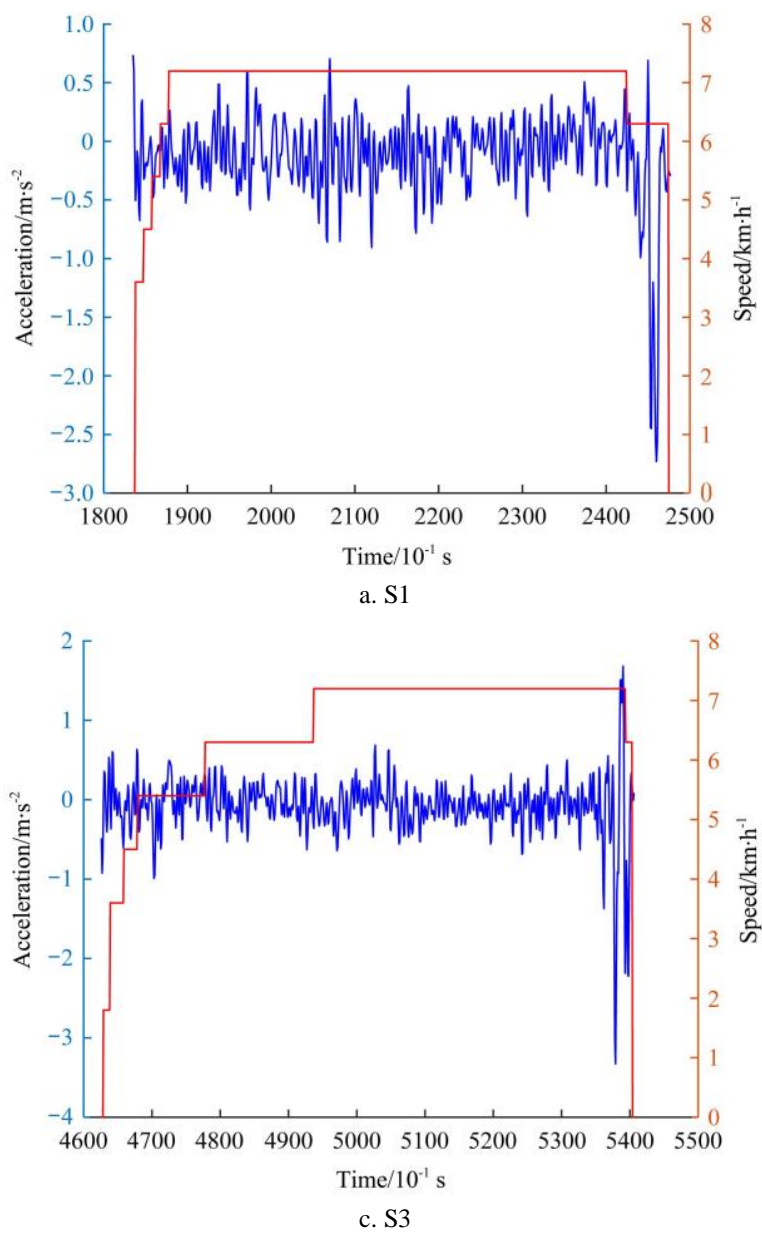

Table 5 Jerk of accelerating and time of acceleration

\begin{tabular}{ccc}
\hline Strips & jerk $/ \mathrm{m}^{3 \cdot \mathrm{s}^{-1}}$ & $R_{02 L} / \%$ \\
\hline S1 & 4.35 & 9.16 \\
S2 & 4.07 & 32.36 \\
S3 & 1.30 & 22.39 \\
S4 & 4.00 & 14.40 \\
S5 & 0.68 & 17.01 \\
S6 & 3.08 & 8.39 \\
S7 & 2.41 & 15.12 \\
\hline
\end{tabular}
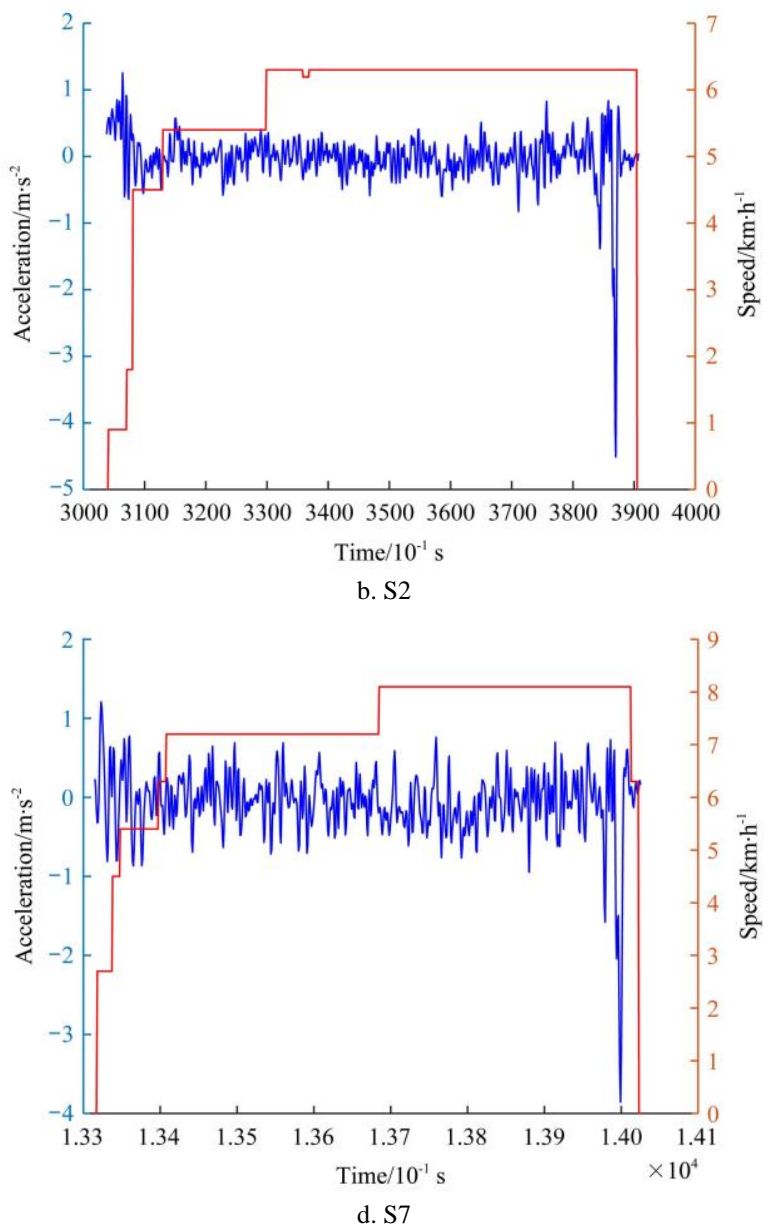

Figure 10 Comparison of accelerated behavior in the starting stage

(2) Operating

Using the methods, as shown in Table 6, the operational characteristics of machinery on each strip can be preliminarily calculated. The mean of speed is the average working speed. The standard deviation of acceleration reflects the smoothness of operating. $\quad T_{\mathrm{S}}$ is the length of working time. $R_{O S}$ reflects the over speed condition. S5, S6, and S7 have the behavior over speed, especially the latter two have serious speeding problems.

Table 6 Technical statistics of operation for Field I

\begin{tabular}{cccccc}
\hline Trips & $\begin{array}{c}\text { Mean of speed } \\
/ \mathrm{km} \cdot \mathrm{h}^{-1}\end{array}$ & $\begin{array}{c}\text { STD of acceleration } \\
/ \mathrm{m}^{-2} \mathrm{~s}^{-2}\end{array}$ & $T_{\mathrm{s}} / \mathrm{s}$ & $R_{L 2 H} / \%$ & $R_{O S} / \%$ \\
\hline S1 & 7.15 & 0.20 & 63.70 & 95.3 & 0 \\
S2 & 5.94 & 0.70 & 86.40 & 70.1 & 0 \\
S3 & 6.68 & 0.75 & 77.50 & 80.8 & 0 \\
S4 & 6.98 & 0.60 & 73.50 & 87.9 & 0 \\
S5 & 6.93 & 0.71 & 79.40 & 80.1 & 2.5 \\
S6 & 7.67 & 0.45 & 68.60 & 43.6 & 52.2 \\
S7 & 7.48 & 0.76 & 70.50 & 42.1 & 46.5 \\
\hline
\end{tabular}

Figure 11 reflects the operating continuity of field II. In the actual operation, there are 11 operation strips along the east-west direction, but due to the interruption, a total of 16 east-west strips are extracted. Therefore, some of the strips in field II have bad continuity.

(3) U-turn

The length of time of U-turn is directly related to the turning radius and distance, turning skill of the operator, time for filling production material, and slack during work. The green circle in Figure 9 is the length of time for the operator to make U-turn in Field I. The larger the circle, the longer the time range of U-turn. Among the seven U-turns, the maximum of time range is $334.6 \mathrm{~s}$, the minimum of time range is $26.7 \mathrm{~s}$, the average of time range is $116.48 \mathrm{~s}$, and the standard deviation of time range is $112.9 \mathrm{~s}$, which means that the same operator has a large difference of U-turn. Moreover, in the $3^{\text {rd }} \mathrm{U}$-turn and the $4^{\text {th }} \mathrm{U}$-turn, the trajectory with zero speed occupies about $75 \%$ of the total time. Obviously, this reflects the operation efficiency.

(4) Stalling and idling

Table 7 shows the stalling and idling in the field I. The total waiting time is $634.1 \mathrm{~s}$, which all come from the idling time. The 


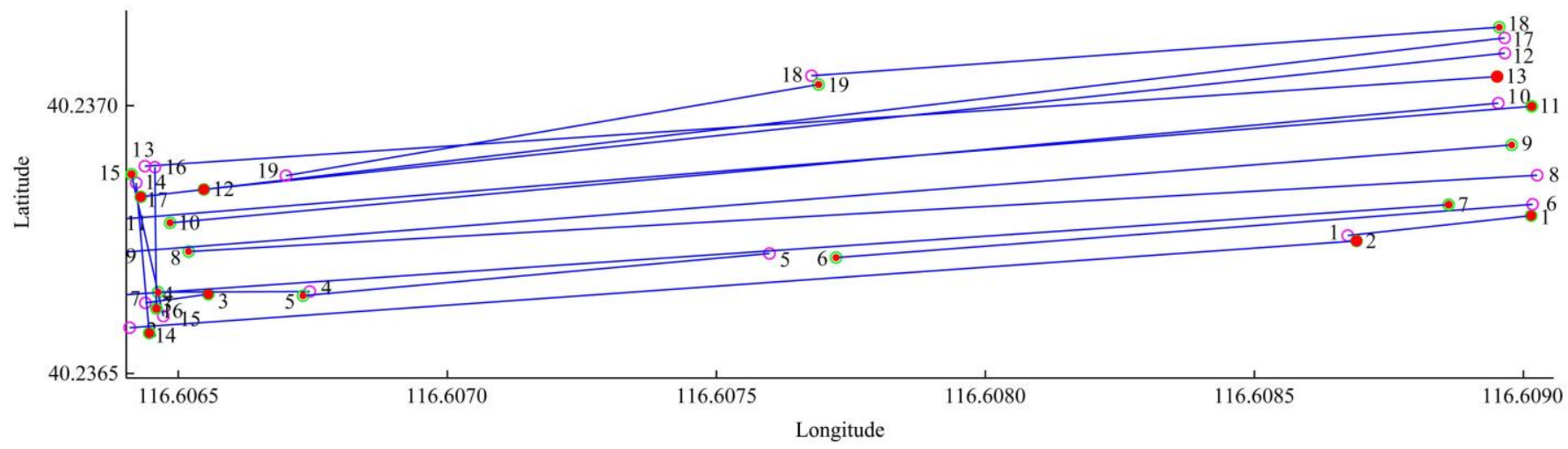

Figure 11 Continuity of operating of field II

Table 7 Statistic of stalling and idling behavior

\begin{tabular}{cccc}
\hline \multirow{3}{*}{ States } & \multicolumn{3}{c}{ Time } \\
\cline { 2 - 4 } & $\leq 10 \mathrm{~s}$ & $\leq 20 \mathrm{~s}$ & $>20 \mathrm{~s}$ \\
\hline Stalling & 0 & 0 & 0 \\
Idling & 58 & 0 & 5 \\
\hline
\end{tabular}

\section{Conclusions}

A smartphone-based solution was proposed to construct the behavior sensing model of agricultural machinery operation to provide technical statistics report for agricultural machinery cooperatives. Three embedded sensors of smartphone including the GNSS, the accelerometer, and the microphone, were used to conduct trajectory segmentation and behavior identification. Field experiments were carried out to extract operating behavior's features and collect operation data during forage maize sowing for case study. Four stages of agricultural machinery operation can be preliminarily segmented by using GNSS speed. Further, the subtle features of sudden acceleration, stalling, idling, and goosing with extra information of acceleration and sound intensity can be extracted. The results show that the detail information of operating behavior can be captured through smartphone's sensors, and the behavior models provide the protocol for in-field operation assessment.

In the future research, it is proposed to evaluate the current threshold of key behaviors through investigation and research, and to provide an evaluation system to achieve online accessing and scoring.

\section{Acknowledgements}

We acknowledge that this research was financially supported by National Key Research and Development Program of China (No. 2016YFB0501805), project of Application of New Mode of Remote Operation and Maintenance Service for Modern Farm Machinery and Equipment, Chinese Universities Scientific Fund (No. 2018XD003).

\section{[References]}

[1] He P, Li J, Wang X. Wheat harvest schedule model for agricultural machinery cooperatives considering fragmental farmlands. Computers and Electronics in Agriculture, 2018; 145: 226-234.

[2] Li E, Yang M, Cook M L. Agricultural machinery cooperatives in china: Origin, development, and innovation. ASABE Annual International Meeting, Reno, NV, United states, June 21-24, 2009; pp.5835-5853.

[3] Zhang Y, Huang Z H. Identifying risks inherent in farmer cooperatives in China. China Agricultural Economic Review, 2014; 6(2): 335-354. (in

\section{Chinese)}

[4] Bochtis D D, Sørensen C G C, Busato P. Advances in agricultural machinery management: A review. Biosystems Engineering, 2014; 126: 69-81.

[5] Stecca G, Baffo I, Galiano G, Clemente F. Design of a holonic remote monitoring and diagnosis system for fleet management. Measurement: Journal of the International Measurement Confederation, 2013; 46(6): 1947-1956.

[6] Orfanou A, Busato P, Bochtis D D, Edwards G, Pavlou D, Sorensen C G, Berruto R. Scheduling for machinery fleets in biomass multiple-field operations. Computers and Electronics in Agriculture, 2013; 94: 12-19.

[7] Lee C J, Kim H J, Ha J W, Cho B J, Choi D S. An isobus-networked electronic self-leveling controller for the front-end loader of an agricultural tractor. Applied Engineering In Agriculture, 2017; 33(6): 757-767.

[8] Al-Aani F S, Darr M J, Covington B R, Powell L J. The performance of farm tractors as reported by can-bus messages. ASABE Annual International Meeting, Orlando, FL, United states, July 17-20, 2016.

[9] Caffaro F, Roccato M, Micheletti Cremasco M, Cavallo E. Falls from agricultural machinery: Risk factors related to work experience, worked hours, and operators behavior. Human Factors, 2018; 60(1): 20-30.

[10] Kou Z H, Wu C C. Smartphone based operating behaviour modelling of agricultural machinery. IFAC-PapersOnLine, 2018; 51(17): 521-525.

[11] Wu C C, Zhou L, Wang J, Cai Y P. Smartphone based precise monitoring method for farm operation. Int $\mathrm{J}$ Agric \& Biol Eng, 2016; 9(3): 111-121.

[12] Bognatz S R. Transient speed vibration analysis - insights into machinery behavior. 62nd Meeting of the Society for Machinery Failure Prevention Technology, MFPT, Virginia Beach, VA, United States, April 6-8, 2008.

[13] Ignatov A D, Strijov V V. Human activity recognition using quasiperiodic time series collected from a single tri-axial accelerometer. Multimedia Tools and Applications, 2016; 75(12): 7257-7270.

[14] Kwon Y, Kang K, Bae C. Unsupervised learning for human activity recognition using smartphone sensors. Expert Systems with Applications, 2014; 41(14): 6067-6074.

[15] Saha J, Chowdhury C, Biswas S. Two phase ensemble classifier for smartphone based human activity recognition independent of hardware configuration and usage behaviour. Microsystem Technologies, 2018; 24(6): 2737-2752.

[16] Hassan M M, Uddin M Z, Mohamed A, Almogren A. A robust human activity recognition system using smartphone sensors and deep learning. Future Generation Computer Systems, 2018; 81: 307-313.

[17] Lee K, Kwan M-P. Physical activity classification in free-living conditions using smartphone accelerometer data and exploration of predicted results. Computers Environment And Urban Systems, 2018; 67: 124-131.

[18] Garcia-Ceja E, Galvan-Tejada C E, Brena R. Multi-view stacking for activity recognition with sound and accelerometer data. Information Fusion, 2018; 40: 45-56.

[19] Vanwye W R, Hoover D L. Management of a patient's gait abnormality using smartphone technology in-clinic for improved qualitative analysis: A case report. Physiotherapy Theory and Practice, 2018; 34(5): 403-410.

[20] Sun R P, Sosnoff J J. Novel sensing technology in fall risk assessment in older adults: a systematic review. Bmc Geriatrics, 2018; 18: 14

[21] Zokas B, Lukoeviius M Human sport activities recognition and registration from portable device. 2018 Symposium for Young Scientists in 
Technology, Engineering and Mathematics (SYSTEM 2018), CEUR-WS, Gliwice, Poland, May 28, 2018; pp.61-65.

[22] Hemalatha C S, Vaidehi V. Associative classification based human activity recognition and fall detection using accelerometer. International Journal of Intelligent Information Technologies, 2013; 9(3): 20-37.

[23] Castignani G, Derrmann T, Frank R, Engel T. Smartphone-based adaptive driving maneuver detection: A large-scale evaluation study. Ieee Transactions on Intelligent Transportation Systems, 2017; 18(9): 2330-2339.

[24] Castignani G, Derrmann T, Frank R, Engel T. Driver behavior profiling using smartphones: A low-cost platform for driver monitoring. IEEE Intelligent Transportation Systems Magazine, 2015; 7(1): 91-102.

[25] Osafune T, Takahashi T, Kiyama N, Sobue T, Yamaguchi H, Higashino T. Analysis of accident risks from driving behaviors. International Journal of Intelligent Transportation Systems Research, 2017; 15(3): 192-202.

[26] Eren H, Makinist S, Akin E, Yilmaz A. Estimating driving behavior by a smartphone. IEEE Intelligent Vehicles Symposium, IV 2012. Institute of Electrical and Electronics Engineers Inc., Alcal de Henares, Madrid, Spain, June 3-7, 2012; pp.234-239.
[27] Johnson D A, Trivedi M M. Driving style recognition using a smartphone as a sensor platform. 14th IEEE International Intelligent Transportation Systems Conference (ITSC 2011), Institute of Electrical and Electronics Engineers Inc., Washington DC, United States, October 5-7, 2011; pp.1609-1615.

[28] Craessaerts G, De Baerdemaeker J, Saeys W. Fault diagnostic systems for agricultural machinery. Biosystems Engineering, 2010; 106(1): 26-36.

[29] Son J-D, Ahn B-H, Ha J-M, Choi B-K. An availability of mems-based accelerometers and current sensors in machinery fault diagnosis. Measurement: Journal of the International Measurement Confederation, 2016; 94: 680-691.

[30] Bietresato M, Friso D, Sartori L. Assessment of the efficiency of tractor transmissions using acceleration tests. Biosystems Engineering, 2012; 112(3): 171-180.

[31] Fadloullah I, Mechaqrane A, Ahaitouf A. Butterworth low pass filter design using evolutionary algorithm, 2017 International Conference on Wireless Technologies, Embedded and Intelligent Systems, WITS, April 19-20, 2017. Institute of Electrical and Electronics Engineers Inc., Fez, Morocco. 